\title{
AUTOCUIDADO DEL ADULTO POST-TRASPLANTE RENAL
}

\author{
Maximiliano Gómez, Rubén Dario Pérez, Adriana Villalobos y Débora Rivillas*
}

\section{Resumen}

Esta investigación describe las características socio-demográficas y los requerimientos de autocuidado del paciente adulto posttrasplante renal, con base en el modelo de enfermería de Dorothea Orem y aporta elementos para el diseño y elaboración de futuros programas educativos. La metodología utilizada fue de tipo cuantitativa con un diseño descriptivo trasversal. La muestra comprende 57 pacientes trasplantados en la E.S.E. Clínica San Pedro Claver de Bogotá D.C. (ISS). Los resultados mostraron una población con edad promedio de 42 años, en su mayoría del sexo femenino, casados, con un nivel educativo primario y secundario, pertenecen a un estrato socio-económico bajo, procedentes de la zona urbana, con múltiples necesidades básicas insatisfechas. Respecto a los requisitos universales de autocuidado, $23 \%$ no cuenta con ingresos necesarios para adquirir los alimentos, $30 \%$ consume una dieta normal y el $25 \%$ practica deportes de alto riesgo. Hay una pobre interacción social y un alto porcentaje no cuenta con elementos de protección personal y prevención de accidentes. En cuanto a los requisitos de autocuidado del desarrollo, se encontró que tienen autoestima alta, dinámica familiar buena, actividad sexual preservada casi siempre sin protección y con un desarrollo acorde con su ciclo vital. En cuanto a la actitud ante la alteración de su estado de salud, 17\% presenta algún tipo de incapacidad, 2\% desconoce los cuidados post-trasplante, $21 \%$ no reconoce los síntoma de rechazo del injerto y $3,5 \%$ no asiste a la unidad cuando presenta complicaciones.

Palabras claves: paciente adulto, trasplante renal, autocuidado, déficit de autocuidado y requisitos de autocuidado.

\section{Introducción}

El trasplante renal es una alternativa de tratamiento del paciente con insuficiencia renal crónica (IRC), que le permite mejorar su calidad de vida y prolongar su supervivencia.

Según datos suministrados por la Red Nacional de Trasplantes y la Corporación Davida, se estima que en Colombia hay cerca de 3.000 personas con IRC en espera de un donante que les permita hacer posible el trasplante. Sin embargo, se presentan factores que limitan su realización debido a los altos costos de la intervención, el tratamiento médico, la escasa oferta de donantes, no contar con un seguro médico y la falta de cobertura de algunos de los medicamentos por el Plan Obligatorio de Salud (POS). A pesar de estos limitantes, la E.S.E. Clínica San Pedro Claver del ISS hasta el año 2002 ha realizado 364 trasplantes renales, con una sobrevida del injerto al primer año de $88 \%$ en caso de donantes vivos y $80 \%$ en donantes

Estudiantes de Postgrado de Enfermería

** Este trabajo fue realizado bajo la tutoría de la Dra. Claudia Patricia Abreo. Grupo de Trasplante de la Clínica San Pedro Claver ISS, Bogotá. cadavéricos. Debido a la complejidad del procedimiento quirúrgico, tipo de donante y tratamiento inmunosupresor, se pueden presentar complicaciones quirúrgicas o médicas. La practica de autocuidado que realice el paciente o su familia es fundamental para el control de factores de riesgo que pueden influir en la aparición de complicaciones postoperatorias.

Fue así como surgió la idea de realizar un estudio descriptivo que reconociera los requerimientos de autocuidado del paciente post-trasplante renal, con el propósito de realizar un aporte documental que permita en el futuro diseñar y elaborar programas educativos.

\section{Metodología}

Se realizó un estudio de tipo cuantitativo descriptivo en el servicio de trasplantes de la E.S.E Clínica San Pedro Claver del ISS. Los participantes fueron pacientes adultos post-trasplante renal, intervenidos en la institución en el período comprendido entre enero de 1990 y junio de 2003. Se tuvieron en cuenta aquellos que asistieron a los controles médicos, cumplieron con los criterios de 
inclusión y desearon participar en forma voluntaria. Mediante un muestreo por conveniencia se seleccionaron 57 pacientes post-trasplante renal, a los cuales se les aplicó una encuesta elaborada con base en el instrumento de valoración de enfermería de Dorothea Orem.

El procedimiento consistió en elaborar una encuesta con el instrumento mencionado, la cual fue revisada por expertos para realizar una prueba piloto. La colecta de la información se efectuó en septiembre de 2003. Se aplicó a los pacientes que asistieron a la reunión mensual del grupo de trasplantados. El total de encuestados fue 80, de los cuales 57 cumplieron con los criterios de inclusión.

La tabulación y el análisis de la información se realizó mediante la ayuda del programa estadístico EPIINFO versión 6.4 y EXCEL 2002, con los cuales se hicieron cálculos estadísticos, gráficas y tablas.

Las consideraciones éticas bajo las cuales estuvo regulado el presente estudio contemplan los principios de beneficencia, justicia y respeto a la dignidad humana, mediante el consentimiento informado.

Las variables para describir los requerimientos de autocuidado fueron: las características socio-demográficas del paciente post-trasplante renal, los requisitos de autocuidado universales, los del desarrollo y aquellos relativos al cambio del estado de su salud.

\section{Resultados}

En cuanto a las características socio-demográficas, el estudio reveló que la edad promedio es 42 años, 58\% son mujeres y $74 \%$ residen en la ciudad. La ocupación o actividad económica es diversa, 26,4\% se dedica al hogar, que va de acuerdo con la mayor proporción del género femenino en el estudio, $21 \%$ son trabajadores independientes, 19,3\% empleados oficiales o privados, 5,3\% realizan estudios, $3,5 \%$ pensionados, $7 \%$ desempleados y $17,5 \%$ no responden (Figura 1).

En cuanto a condiciones socio-económicas, se encontró que el $8,8 \%$ pertenece o se clasifica en el estrato uno, $36,8 \%$ en el dos, $35,1 \%$ en el tres y $5,3 \%$ en el cuatro. El $100 \%$ de los trasplantados cuentan con un seguro médico. El 17,5\% afirma haber sido re-trasplantado. El 60\% de los trasplantados son casados o viven en unión libre. Con respecto al grado de escolaridad, el estudio reveló que el $26 \%$ ha realizado estudios primarios, $44 \%$ básica secundaria, $7 \%$ carrera técnica, $9 \%$ estudios superiores y $14 \%$ no responde (Figura 2).

Dentro de los factores condicionantes básicos, es fundamental el entorno donde se desarrolla el paciente para realizar acciones de autocuidado, así como el lugar de residencia. El estudio reveló que $49 \%$ tienen vivienda propia y $44 \%$ viven en arriendo, $95 \%$ cuenta con agua potable, $95 \%$ tiene energía eléctrica, $75 \%$ conexión de gas natural, $91 \%$ una línea telefónica, $88 \%$ alcantarillado,

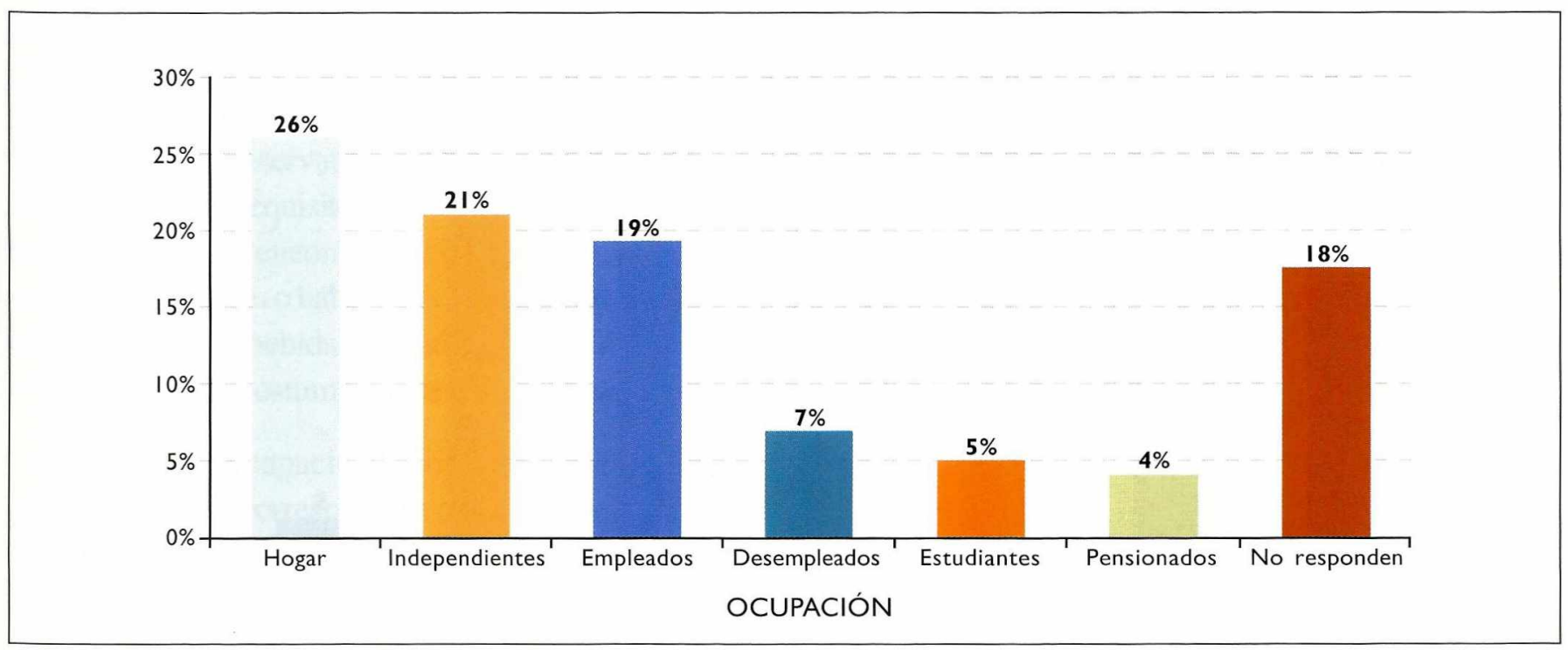

Figura I. Actividad económica de los pacientes trasplantados: hogar, trabajadores independientes, empleados, desempleados, estudiantes, pensionados y no responden. 
$89 \%$ cuenta con servicio de recolección de basuras y el $5 \%$ no responde (Figura 3).

$\mathrm{Al}$ analizar el grupo de variables en cuanto a requisitos de autocuidado universales, el estudio reveló que el $30 \%$ de los trasplantados consume una dieta normal, $7 \%$ dieta hiposódica, $2 \%$ dieta hipograsa, $33 \%$ combinan una hiposódica, hipoglúcida e hipograsa, 3,5\% combina una hiposódica e hipoglúcida, $9 \%$ combina una hipoglúcida e hipograsa. El 7\% una hiposódica, hipoglúcida, hipograsa más restricción de líquidos y el $5 \%$ consume una dieta combinada hiposódica e hipograsa. El 1,5\% no responde (Tabla 1).

Respecto a los ingresos económicos para adquirir los alimentos se encontró que el $70 \%$ cuenta con

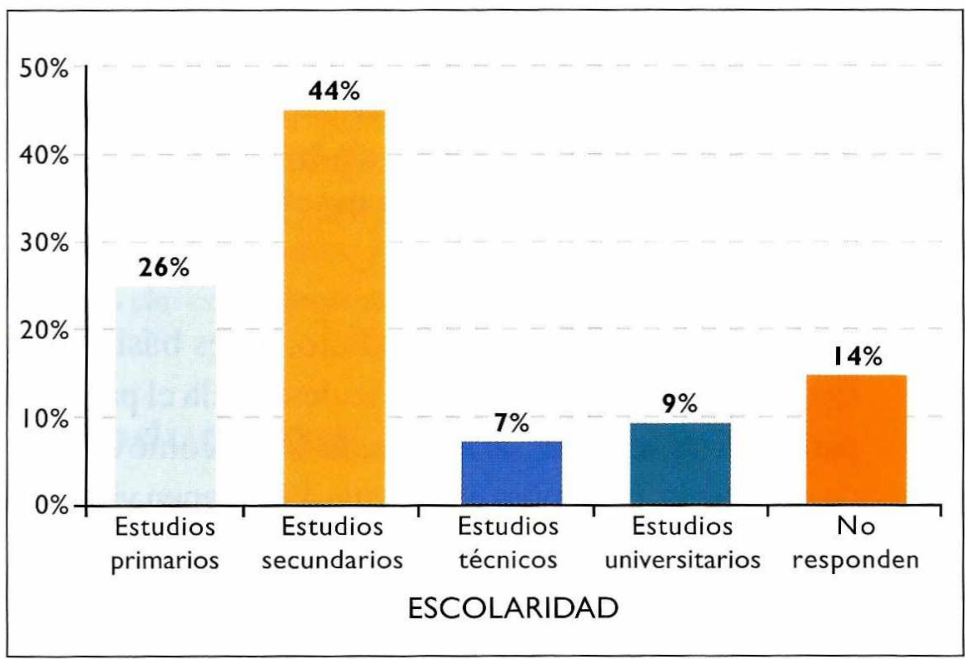

Figura 2. Grado de escolaridad del paciente trasplantado: estudios primarios, secundarios, técnicos, universitarios y no responden. recursos suficientes, mientras que $23 \%$ no, y $7 \%$ no responden.

En relación con problemas en la eliminación y excreción, el estudio reveló que $16 \%$ de los trasplantados presentan estreñimiento, 55\% diarrea, 14 disuria, $2 \%$ hematuria y $7 \%$ disminución del volumen urinario.

Referente a la actividad, se observó que $35 \%$ de los trasplantados no realiza ejercicio físico. Del $65 \%$ restante, $59 \%$ camina, $8 \%$ trota, $14 \%$ realiza ciclismo, $11 \%$ juega fútbol y $8 \%$ realiza aeróbicos (Tabla 2).

Con respecto a los períodos de descanso y sueño diurnos, se halló que 51\% no lo puede hacer.

La interacción social de los pacientes trasplantados es buena. El estudio reveló que $86 \%$ sigue frecuentando y visita amigos. El $67 \%$ no participa en grupos sociales, mientras que $33 \%$ están integrados o pertenecen por lo menos a uno. De ellos, $53 \%$ practica un credo religioso y un $47 \%$ asiste a otros grupos sociales (deportivos, culturales, estudiantil, club social, etc.). Sobre la protección personal y la prevención de accidentes se encontró que el $31,6 \%$ de los trasplantados tiene extintor en la casa o sitio de trabajo, $26 \%$ ha adaptado su casa con pasamanos o barandas en las gradas, $26 \%$ utiliza cinturón de seguridad cuando conduce un vehículo, $28 \%$ usa gafas de seguridad o adaptadas por disminución de la agudeza visual,

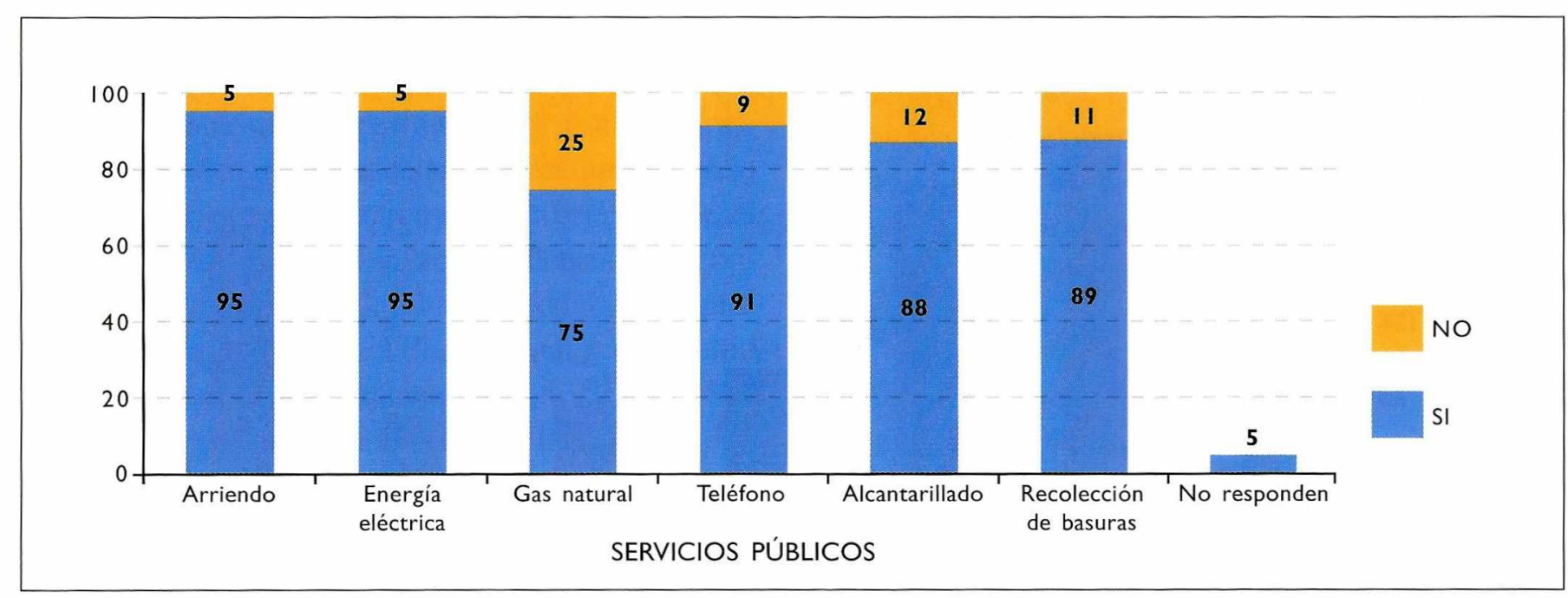

Figura 3. Servicios públicos de la vivienda del paciente trasplantado: arriendo, energía eléctrica, gas natural, teléfono, alcantarillado, recolección de basuras y no responden. 
$17,5 \%$ tiene en cuenta la señalización en el sitio de trabajo o en la calle como peatón y 10,5\% utiliza otros elementos para protegerse.

En el segundo grupo de variables de los requisitos de autocuidado del desarrollo, el estudio reveló que los pacientes trasplantados en un $98 \%$ tienen una alta autoestima, autoconcepto y autoimagen. En cuanto a metas y expectativas futuras, $19 \%$ quiere iniciar o reanudar sus estudios, $3,5 \%$ desea contraer matrimonio y conformar una familia, $2 \%$ tener hijos, $63 \%$ recuperar su estado de salud, 56\% emprender una actividad laboral, $26 \%$ salir de viaje y recrearse y $10,5 \%$ no responde.

La dinámica familiar de los pacientes trasplantados en términos generales es buena, el estudio reveló que en el $88 \%$ hay buenas relaciones intrafamiliares, $9 \%$ son regulares y $3 \%$ no responde. Los principales conflictos familiares son las discusiones (38,6\%), sobreprotección de la familia (9\%), abandono (3,5\%), y dependencia $(10,5 \%)$.

La percepción que tienen de su estado de salud actual, en términos generales es buena. La investigación reveló que $64,9 \%$ refiere tener buena salud, $24,6 \%$ aceptable y $10,5 \%$ regular.

No tiene vida sexual activa $32 \%$, mientras $68 \%$ la conserva; de los últimos $15,4 \%$ se protegen de un embarazo o de una enfermedad de transmisión sexual (ETS) mediante pareja estable más el uso de preservativo, $53,9 \%$ con pareja estable sin protección, $10,3 \%$ no se protegen, $5 \%$ utiliza esterilización (ligadura de trompas) y 15,4\% utiliza preservativo. Con respecto al análisis de la variable de los requisitos de autocuidado ante el cambio en la salud, se encontró que 68,5\% los abandonó o cambió costumbres o hábitos nocivos. El resto consume cigarrillo, (3,5\%) bebidas alcohólicas $(10,5 \%)$ y 17,5\% tiene otro tipo de costumbres dietéticas (Figura 4).

Se hallaron incapacidades o limitaciones en 17\%, de los cuales $7 \%$ corresponde a disminución de la agudeza visual, $8 \%$ a disminución de la agudeza auditiva y $2 \%$ a amputaciones de miembros.

En relación con el conocimiento de los cuidados posttrasplante, el 98\% asegura que los conoce.
En cuanto al conocimiento y a la capacidad para identificar los efectos adversos del tratamiento médico, el $93 \%$ afirma que conoce y distingue los medicamentos y sus efectos adversos, el $3 \%$ niega conocerlos y $5 \%$ no responde. El $98 \%$ afirma que ingiere los medicamentos en la dosis y horario indicado por el médico.

\begin{tabular}{lc}
\multicolumn{2}{c}{$\begin{array}{c}\text { Tabla } \begin{array}{c}\text { Tipo de dieta que consume el } \\
\text { paciente trasplantado }\end{array} \\
\text { Tipo de dieta }\end{array}$} \\
\hline Normal & 30 \\
Hiposódica & 7 \\
Hipograsa & 2 \\
Hiposódica + hipoglúcida + hipograsa & 33 \\
Hiposódica + hipoglúcida & 3,5 \\
Hipoglúcida + hipograsa & 9 \\
Hiposódica + hipoglúcida + hipograsa + & \\
restricción de líquidos & 7 \\
Hiposódica + hipograsa & 7 \\
No responden & 1,5 \\
\hline TOTAL & 100
\end{tabular}

Tabla 2. Realización de actividad y ejercicio físico del paciente trasplantado.

\begin{tabular}{|c|c|}
\hline Actividad y ejercicio físico & Porcentaje\% \\
\hline No & 35 \\
\hline SI & 65 \\
\hline Caminar & 59 \\
\hline Trote & 8 \\
\hline Ciclismo & 14 \\
\hline Fútbol & 11 \\
\hline Aeróbicos & 8 \\
\hline
\end{tabular}

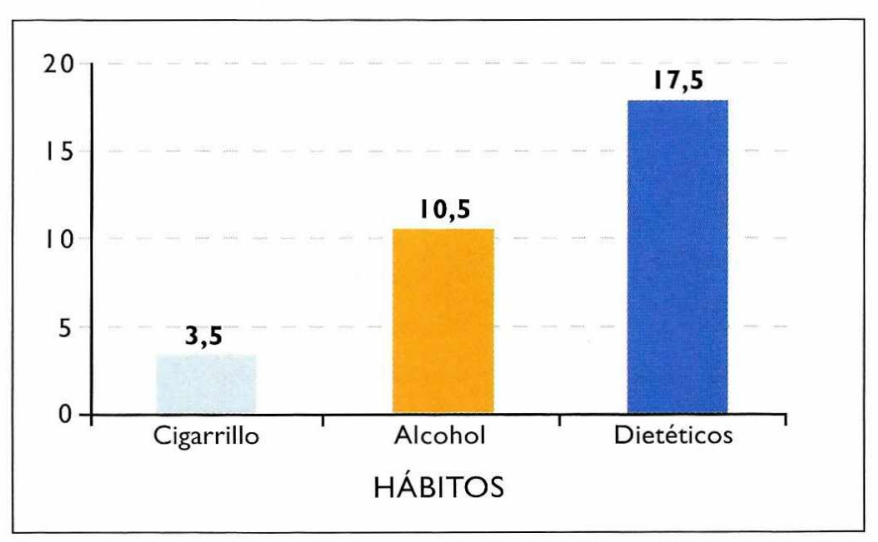

Figura 4. Hábitos nocivos del paciente trasplantado. 
En relación con la identificación y reconocimiento de los síntomas y signos de rechazo, 79\% de los trasplantados los reconocen e identifican, $21 \%$ no y $3,5 \%$ no asisten a urgencias cuando estos síntomas y signos se presentan.

El 96,5\% asiste a los controles programados médicos y de enfermería(Tabla 3).

\section{Discusión}

El estudio mostró que el promedio de edad es 42 años, lo cual indica que la población investigada es madura, con capacidad intelectual, voluntad, intención y conciencia para desarrollar actividades potenciales de autocuidado en una situación específica; sin embargo, al considerar el nivel académico, se observa un alto porcentaje solo con primaria y básica secundaria, que en algunos casos específicos limita el aprendizaje y por ende el desarrollo de las prácticas de autocuidado.

En relación con la disponibilidad, la mayoría se dedica a actividades del hogar, son trabajadores independientes, estudiantes, pensionados o están desempleados; lo cual indica que son pacientes con adecuado tiempo y disposición para proporcionar su cuidado; sin embargo, puede convertirse en una limitante por la falta de suficientes ingresos económicos.

En la procuración del autocuidado hay un porcentaje importante que presenta conductas o costumbres nocivas para su bienestar, como el consumo de cigarrillo y alcohol.

Tabla 3. Conocimientos de los cuidados y tratamiento médico del paciente trasplantado

\begin{tabular}{lcc} 
Variable & $\begin{array}{c}\text { Sí } \\
\text { conoce } \%\end{array}$ & $\begin{array}{c}\text { No } \\
\text { conoce \% }\end{array}$ \\
\hline Cuidados post-trasplante & 98 & 2 \\
\hline Medicamentos y efectos adversos & 93 & 7 \\
\hline $\begin{array}{l}\text { Cumplimiento de horarios y } \\
\text { dosis de los medicamentos }\end{array}$ & 98 & 2 \\
\hline $\begin{array}{l}\text { Asistencia a controles médicos } \\
\text { y de enfermería }\end{array}$ & 96,5 & 3,5 \\
\hline
\end{tabular}

Asistencia a urgencias cuando se presentan síntomas

96,5 3,5
En un número bajo hay limitaciones visuales y auditivas o amputaciones, algunos no asisten a los controles y no están preparados para proveer su autocuidado. La población estudiada es variable, procedente en su mayor parte de la zona urbana y con predominio de las clases baja y media baja, caracterizada por poseer costumbres propias de su entorno. La gran mayoría han modificado su estilo de vida y han optado conductas saludables indicadas por el equipo médico y que se ajustan a su estado de salud. Sin embargo muchos siguen desarrollando acciones nocivas para su bienestar y se exponen a riesgos que podrían llegar afectar la sobrevida del injerto. Se debe por lo regular a que el paciente se siente bien y quiere seguir el estilo de vida que llevaba antes de enfermarse.

El estudio no contempló una variable que nos permitiera conocer a fondo los cuidados o las experiencias de autocuidado de los pacientes trasplantados, pero en términos generales se puede inferir que aquellos con insuficiencia renal crónica o aguda se les educa para que sigan determinados cuidados propios de la patología, muchos de los cuales se mantienen o se modifican después del trasplante.

La mayoría de los trasplantados cuentan con los requisitos universales o los materiales esenciales para la vida como el agua, el aire y los alimentos; sin embargo, un porcentaje considerable no tiene acceso a los servicios públicos y sus ingresos económicos no son suficientes para una alimentación adecuada.

En cuanto a las funciones excretoras, un número importante presenta problemas; las complicaciones más frecuentes son las infecciosas debidas a microorganismos oportunistas, por la inmunosupresión, que causan diarreas e infecciones de las vías urinarias. que llevan a presentar diarreas, estreñimiento, síndromes de mala absorción e infecciones de las vías urinarias. En cuanto a los riesgos, un pequeño grupo utiliza algunas medidas de protección y de prevención de accidentes, pero la mayoría no lo hace exponiendo su integridad y aumentando la posibilidad de sufrir un accidente.

Un porcentaje considerable desconoce los cuidados post-trasplante, el tratamiento médico, así como los síntomas y signos de rechazo del injerto. Se relaciona con las condiciones socio-económicas precarias, un nivel 
educativo básico y a la falta de voluntad para realizarlos, aumentando el riesgo de complicaciones que comprometan la sobrevida del injerto, lo cual se demuestra en el estudio por el alto porcentaje de re-trasplante. Respecto a los resultados del resto de variables estudiadas, se puede inferir que en cuanto a la seguridad social, la totalidad de los trasplantados cuentan con un seguro médico que garantiza la continuidad del tratamiento.

La mayoría de pacientes trasplantados tiene un hogar estable que exige una responsabilidad y de alguna manera cuenta con un compañero (a) que le colabora en su cuidado. En algunos casos ese tipo de responsabilidad (la búsqueda de recursos) desplaza la disponibilidad para su autocuidado, lo cual puede influir en el bienestar del paciente.

La mayoría de los trasplantados no tiene capacidad económica para adquirir vivienda, como lo muestran los resultados frente al nivel socio-económico que los clasifica dentro de las clases baja y media baja.

Respecto a los ingresos económicos, un porcentaje significativo no cuenta con los necesarios para adquirir los alimentos, tornándose en un factor de riesgo para el paciente trasplantado al no haber un buen aporte nutricional, que asociado al tratamiento inmunosupresor puede aumentar la posibilidad de infecciones o alteraciones metabólicas que pueden influir en la sobrevida del injerto.

A los pacientes trasplantados se les recomienda la actividad física con ejercicios pasivos, no realizar deportes de contacto o de alto riesgo, para evitar traumatismos en el sitio del injerto. Sin embargo los resultados nos muestran un alto porcentaje de sedentarismo y unos pocos realizan actividades deportivas, inclusive aquellos de contacto que pueden causar trauma abdominal y afectar el injerto.

En cuanto al descanso, la mayor parte no lo hacen en las horas diurnas por su actividad económica u ocupación ya sea independiente, dedicado al hogar o empleado.

La interacción social y la sociedad juegan un papel importante en el aprendizaje del autocuidado. Muchas de las acciones han sido aprendidas en el entorno de la cultura de cada individuo y si lo analizamos en nuestros casos vemos como un buen número se han aislado o marginado de su entorno. Cabe recodar que la sociedad constituye una red de apoyo de gran importancia. Al analizar las variables de los requisitos del desarrollo, se encontró que los trasplantados tienen un alto grado de valoración de sí mismos, que permite continuidad del tratamiento y facilita que el paciente tenga voluntad y procuración para la provisión de su cuidado. Es muy importante que en la valoración psicológica y psiquiátrica pre-trasplante se descarten conductas sociopáticas.

La dinámica familiar de los trasplantados es fundamental, porque es su principal apoyo. Sin embargo, cuando hay sobreprotección, se inhabilita el autocuidado y se crea un estadio de dependencia como lo vemos en los resultados del estudio. Cuando esto sucede, la familia o el cuidador termina cansándose y en muchos casos abandona al paciente.

Un alto porcentaje dice estar en buen estado de salud, pues para un paciente terminal sometido a la terapia dialítica, el trasplante se convierte en un renacer, es volver a vivir y su calidad de vida mejora. En muchos de los casos los trasplantados tienen patologías asociadas que interfieren su estado de salud, por lo cual algunos refieren estar en regular o mal estado. La mayoría de trasplantados conservan la actividad sexual, sin embargo muchos no se protegen de una ETS o de un embarazo. En el caso de las mujeres trasplantadas, una de las recomendaciones es no tener hijos debido a las potenciales complicaciones que la gestación puede desencadenar, en especial en el sistema inmunológico y los posibles efectos del tratamiento para el feto. De igual manera, para el hombre una ETS puede acarrear problemas de difícil manejo.

Un alto porcentaje de trasplantados practica costumbres nocivas para la salud, que posiblemente vienen desde antes de presentar la insuficiencia renal o que por el hecho de sentirse bien después del trasplante las hayan iniciado. Pueden causar los mismos efectos nocivos que en cualquier individuo o se pueden potencializar, pero sería tema de otra investigación. Esto nos indica alguna falla en la evaluación psicológica pre-trasplante o en el proceso educativo post-trasplante.

Las incapacidades o limitaciones se presentan en bajo porcentaje y se convierten en factor desfavorable en el 
proceso de autocuidado, sobretodo las visuales y auditivas, que pueden llevar a que el cuidado sea asumido por una segunda persona, creando un estado de dependencia que genera problemas intrafamiliares y afecta la continuidad del tratamiento.

\section{Conclusiones}

Los requisitos universales del autocuidado son comunes para todos los seres humanos. Corresponden a las acciones humanas que proporcionan condiciones internas y externas para mantener la estructura y la actividad, que a su vez apoyan el desarrollo y la maduración humana. Cuando se proporciona en forma eficaz, el autocuidado fomenta en forma positiva la salud y el bienestar. El estudio mostró que un porcentaje significativo de los pacientes trasplantados carecen de estos requisitos y como consecuencia se presenta un déficit de autocuidado.

Los requisitos en los pacientes trasplantados están asociados con los procesos de desarrollo humano, así como a las condiciones y eventos que ocurren durante diversos estadíos del ciclo vital. Promueven procedimientos para la vida y para la maduración y previenen las circunstancias perjudiciales o mitigan sus efectos.

El profesional de enfermería juega un papel importante en el proceso de educativo durante el pre y post-trasplante renal, convirtiéndose en guía y maestro que permite modificar conductas nocivas adquiridas y adoptar comportamientos saludables.

\section{Recomendaciones}

Para futuras investigaciones en autocuidado se hace necesario diseñar y validar un instrumento que garantice al investigador confiabilidad en la información. El abordaje del paciente se debe realizar en el entorno y medio en el cual interactúa. Es importante realizar proyectos de tipo cuantitativo y cualitativo que permitan conocer las prácticas y experiencias de autocuidado del paciente trasplantado. Producto de estas investigaciones será diseñar y estructurar un programa de educación continua dirigido al paciente durante las fases de pre y post-trasplante, orientado hacia la modificación de costumbres y hábitos nocivos, para adoptar conductas saludables y habilitar al paciente para un nuevo estilo de vida.

\section{Lecturas recomendadas}

- Abreo C P. Manual de cuidados post-trasplante. Grupo de Trasplante Clínica San Pedro Claver. Bogotá: ISS, 2000.

- Borrero, J, Montero, O. Fundamentos de Medicina. Nefrología. 4 ed. Medellín: Corporación para Investigaciones Biológicas 2003.

- Borrero J, y Cala H: Roso A. Fundamentos de Medicina. Nefrología.3 ed. Medellín: Presencia 1993.

- Douglas J N. Trasplante Renal. Cuidados del paciente renal. 1988.

- Henrrich, W L. Diálisis. 2 ed, México Mc Graw-Hill Interamericana, 2001.

- Martínez MJ. Guías de Manejo para la unidad renal. Bucaramanga: Universidad Industrial de Santander, 2000.

- Orem D E. Nursing concepts of practice. 6 th ed. St Louis, Missouri: Mosby, 2001 p.97-120.

- Polit H. Investigación científica en ciencias de la salud. 6 ed. Mexico: McGraw - Hill Interamericana 2000.

- Red Nacional de Trasplante. Disponible http// www.Redbogota.org

- Saenz M. Nuevos enfoques de educación para la salud. Atención Primaria. Bogotá:1998. p 13.

- Sellarés, VL. y otros. Manual de Nefrología Clínica. Diálisis y Trasplante Renal. Madrid: Harcourt Broce, 1997.

- Tomey, A M. Modelos y teorías en enfermería. 3 ed. Madrid: Mosby Doyma, 1994 p 247-325.

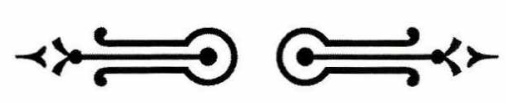

